\title{
Convergent non complete interpolatory quadrature rules
}

\author{
U. Fidalgo and J. Olson
}

Abstract We find a family of convergent schemes of nodes for non-complete interpolatory quadrature rules.

\section{Introduction}

Let $C([-1,1])$ be the set of all continuous functions defined on $[-1,1]$. Given an $n$-tuple of nodes $\mathbf{x}_{n}=\left(x_{1, n}, \ldots, x_{j, n}\right)$ satisfying $-1<x_{1, n}<x_{2, n}<\cdots<x_{n, n}<1$, we consider integration rules

$$
I_{n}[f]=\sum_{j=1}^{n} w_{j, n} f\left(x_{j, n}\right), \quad f \in C[-1,1]
$$

associated to the integrals

$$
I(f)=\int_{-1}^{1} f(x) \mathrm{d} \lambda_{0}(x), \quad \text { where } \quad \frac{\mathrm{d} \lambda_{0}(x)}{\mathrm{d} x}=\frac{1}{\pi \sqrt{1-x^{2}}} .
$$

The numbers $w_{j, n}, j=1, \ldots, n$ are called weights.

An integration rule $I_{n}[\cdot]$ is said to be interpolatory if there exists a number $m \in\{0,1, \ldots, 2 n-1\}$, such that the following equality holds for every polynomial $p$ with degree $\leq m$ (we denote $p \in \Pi_{m}$ ):

Ulises Fidalgo

Department of Mathematics, Applied Mathematics, and Statistics, Case Western Reserve University, Cleveland, Ohio 43403, e-mail: uxf6@case. edu This author's research was supported in part by the research grant MTM2012-36372-C03-01 funded by "Ministerio de Economía y Competitividad", Spain.

Jacob Olson

Case Western Reserve University, Cleveland, Ohio 43403 e-mail: jfo27@case.edu 


$$
I_{n}[p]=\int_{-1}^{1} p(x) \mathrm{d} \lambda_{0}(x) .
$$

When the equality (3) holds for certain $m$, and is not extendable for all polynomials with degree $m+1$, we say that $I_{n}[\cdot]$ is an interpolatory quadrature rule with $m$-degree of exactness. When $m=2 n-1 I_{n}$ is the Gaussian quadrature rule.

Consider a sequence of interpolatory quadratures $\left\{I_{n}\right\}_{n \in \Lambda}$ constructed with the following schemes of nodes and weights

$$
\mathbf{x}=\left\{\mathbf{x}_{n}=\left(x_{1, n}, \ldots, x_{n, n}\right)\right\}_{n \in \Lambda} \quad \text { and } \quad \mathbf{w}=\left\{\mathbf{w}_{n}=\left(w_{1, n}, \ldots, w_{n, n}\right)\right\}_{n \in \Lambda},
$$

respectively.

We say that $\left\{I_{n}\right\}_{n \in \Lambda}$ is convergent if

$$
\lim _{n \in \Lambda} I_{n}[f]=\int_{-1}^{1} f(x) \mathrm{d} \lambda_{0}(x), \quad \text { for all } f \in C[-1,1] .
$$

According to a classical result of Pólya [5, page 130], when $m(n) \rightarrow \infty$ as $n \rightarrow \infty$, the equality (5) holds true if and only if $\sup _{n \in \Lambda} \sum_{j=1}^{n}\left|w_{j, n}\right|<\infty$. This condition is satisfied if the weights $w_{j, n}$ are all positive. From (3) we observe that

$$
\sum_{j=1}^{n} w_{j, n}=\int_{-1}^{1} \frac{\mathrm{d} x}{\pi \sqrt{1-x^{2}}}=1<\infty .
$$

In the Gaussian quadrature rule (maximum degree of exactness $m(n)=2 n-1$ ) the weights $w_{j, n}, j=1, \ldots, n$ are all positive and the convergence of the rule is guarantied. However the nodes are all fixed. For each $n \in \mathbb{N}$ the points of evaluation $x_{j, n}, j=1, \ldots, n$ must be the roots of the $n$th orthogonal polynomial with respect to $\lambda_{0}$ (see for instance [19]). This is the Chebyshev polynomial with degree $n$. This means that if we do not have the value of $f$ at each point $x_{j, n}$ the calculus gets stuck. It is convenient to have more flexibility in the distribution of the evaluation nodes. We study convergent interpolatory integration rules with orders of exactness $m<2 n-1$.

The authors of [3] analyze a wide class of interpolatory quadrature rules with $m(n)$ degrees of exactness behaving as follows

$$
\lim _{n \rightarrow \infty} \frac{m(n)}{2 n}=a \in[0,1]
$$

They characterized all possible weak*-limit points of the sequence of counting measures associated with distribution of nodes corresponding to a convergent scheme.

A sequence $\left\{v_{n}\right\}_{n \in \mathbb{N}}$ of measures is said to converge weakly to the measure $v$ provided that there exists a compact set $K$ containing the support of $v$ and of each $v_{n}$, and that 


$$
\lim _{n \in \mathbb{N}} \int f d v_{n}=\int f d v
$$

for each continuous function $f$ on $K$. In such a case, we write $v_{n} \stackrel{*}{\rightarrow} v$. We say that $v$ is a weak*-limit of the sequence $\left\{\eta_{n}\right\}_{n \in \mathbb{N}}$ if some subsequence of $\left\{v_{n}\right\}_{n \in \Lambda \subset \mathbb{N}}$ is weakly convergent to $v$.

Set two schemes of numbers as in (4) associated to an interpolatory quadrature rule $\left\{I_{n}\right\}_{n \in \Gamma}$ where the degree of exactness satisfies $(7)$ for certain $a \in[0,1]$. We also consider its corresponding sequence $\left\{\eta_{n}\right\}_{n \in \mathbb{N}}$ of probability counting measures

$$
\eta_{n}:=\frac{1}{n} \sum_{j=1}^{n} \delta_{x_{j, n}}, \quad n \in \mathbb{N}
$$

According to [2], if the rule $\left\{I_{n}\right\}_{n \in \Lambda}$ is convergent then every weak*-limit $v$ of the the sequence $\left\{\eta_{n}\right\}_{n \in \mathbb{N}}$ satisfies that

$$
v \geq a \lambda_{0} .
$$

Also from [2] we have that this necessary condition is not sufficient. Theorem 1 states conditions of convergence on the distribution of nodes.

Let us introduce some previous notation. Set $\mathcal{K}_{1}$ and $\mathcal{K}_{2}$ two compact subsets of the complex plane $\mathbb{C}$. Let $\operatorname{dist}\left(\mathcal{K}_{1}, \mathcal{K}_{2}\right)=\min \left\{|| x-y \mid \|: x \in \mathcal{K}_{1}\right.$ and $\left.y \in \mathcal{K}_{2}\right\}$ denote the distance between $\mathcal{K}_{1}$ and $\mathcal{K}_{2}$. Consider a compact set $K \subset \mathbb{C} \backslash[-1,1]$, and a measure $\mu$ supported on $K$. A measure $\widetilde{\mu}$ supported on $[-1,1]$ is said to be the balayage of $\mu$ if they have the same total variation $\|\mu\|=\|\widetilde{\mu}\|$ and their logarithmic potentials coincide on $[-1,1]$. This is

$$
V^{\tilde{\mu}}(x)=\int \log \frac{1}{|x-t|} \mathrm{d} \widetilde{\mu}(t)=\int \log \frac{1}{|x-\zeta|} \mathrm{d} \mu(\zeta)=V^{\mu}(x), \quad x \in[-1,1] .
$$

In [17, Section II.4] we can find a deep study about balayage of measures. We are now ready to state the main result of this paper:

Theorem 1. Fix a number $\kappa \in \mathbb{N}$ and a probability discrete measure

$$
\sigma=\frac{1}{\kappa} \sum_{k=1}^{\kappa} \delta_{\zeta_{k}}, \quad \zeta_{k} \subset \mathbb{C} \backslash[-1,1], \quad k=1, \ldots, \kappa .
$$

Assume that $\sigma$ is symmetric with respect to $\mathbb{R}$ with $\operatorname{dist}\left(\left\{\zeta_{1}, \ldots, \zeta_{\kappa}\right\},[-1,1]\right)>1$. Denote $\widetilde{\sigma}$ the balayage measure associated to $\sigma$ supported on the interval $[-1,1]$. Given a rational number $a \in[0,1]$, consider a subsequence $\Lambda \subset \mathbb{N}$ such that for each $n \in \Lambda, 2 \frac{1-a}{\kappa} n \in \mathbb{N}$. Let $\mathbf{x}=\left\{\mathbf{x}_{n}=\left(x_{1, n}, \ldots, x_{n, n}\right)\right\}_{n \in \Lambda}$ be a scheme of nodes. If for each $j=1, \ldots n, n \in \Lambda$ there are two constants $A \geq 0$ and $\ell>0$ satisfying

$$
\left|(1-a) \pi \int_{x_{j, n}}^{1} \mathrm{~d} \widetilde{\sigma}(t)-a \arccos x_{j, n}-\frac{2 j-1}{2 n} \pi\right| \leq A e^{-\ell n}
$$


then there always exist weights $\mathbf{w}=\left\{\mathbf{w}_{n}=\left(w_{1, n}, \ldots, w_{n, n}\right)\right\}_{n \in \mathbb{N}}$, where $\left\{I_{n}\right\}_{n \in \Lambda}$ corresponding to $\mathbf{x}$ and $\mathbf{w}$ is convergent.

In Section 2 we give some explicit schemes that satisfy the relation (10). The statement of Theorem 1 is proved in Section 5 In such proof we use results coming from the orthogonal polynomials theory that are analyzed in Section 3 and Section 4 In Section 3 we study algebraic properties of families of orthogonal polynomials and their connections with convergent conditions of non-complete interpolatory quadrature rules. In Section 4 we describe the strong asymptotic behavior of an appropriated family of orthogonal polynomials with respect to a varying measure.

\section{Some explicit convergent schemes of nodes}

We consider three particular cases where the inequality (10) holds. In the three situations the measure $\sigma=\delta_{\zeta}$ corresponds to a Dirac delta supported on a point belonging to the real line $\zeta>2$. Hence the situations are when $a$ takes the values 0 , $1 / 2$, and 1 .

According to [17, Section II.4 equation (4.46)], the balayage measure of $\sigma=\delta_{\zeta}$ on $[-1,1]$ has the following differential form

$$
\mathrm{d} \widetilde{\sigma}(t)=\frac{\sqrt{\zeta^{2}-1}}{\pi(\zeta-t) \sqrt{1-t^{2}}} \mathrm{~d} t .
$$

We study the function

$$
I_{a}(x)=(1-a) \pi \int_{x}^{1} \mathrm{~d} \widetilde{\sigma}(t)=(1-a) \sqrt{\zeta^{2}-1} \int_{x}^{1} \frac{\mathrm{d} t}{(\zeta-t) \sqrt{1-t^{2}}} .
$$

Taking the change of variables $t=\cos \theta$ and taking into account $\zeta>2(\varphi(\zeta)>2$ implies that $\arg (1-\varphi(\zeta))=\pi)$, we have that

$$
I_{a}(x)=(1-a)\left(-\arccos x+2 \arg \left(e^{i \arccos x}-\varphi(\zeta)\right)-2 \pi\right) .
$$

In this situation the condition of convergence (10) in Theorem 1 acquires the following form

$$
\left|\arccos x_{j, n}+2(1-a)\left[\pi-\arg \left(e^{i \arccos x}-\varphi(\zeta)\right)\right]+\frac{2 j-1}{2 n} \pi\right| \leq A e^{-\ell n} .
$$

Then a scheme $\mathbf{x}=\left\{\mathbf{x}_{n}=\left(x_{1, n}, \ldots, x_{n, n}\right)\right\}_{n \in \Lambda}$ that satisfies the following relation is convergent

$$
\arccos x_{j, n}+2(1-a)\left[\pi-\arg \left(e^{i \arccos x}-\varphi(\zeta)\right)\right]=-\frac{2 j-1}{2 n} \pi-A e^{-\ell n}=\kappa_{j, n},
$$


with $A>0$ and $\ell>0$. This means that

$$
\cos \left(\arccos x_{j, n}+2(1-a)\left[\pi-\arg \left(e^{i \arccos x}-\varphi(\zeta)\right)\right]\right)=\cos \kappa_{j, n}
$$

Using the cosine addition formula we have that

$$
\begin{gathered}
x_{j, n} \cos \left\{2(1-a)\left[\pi-\arg \left(e^{i \arccos x}-\varphi(\zeta)\right)\right]\right\} \\
-\sqrt{1-x_{j, n}^{2}} \sin \left\{2(1-a)\left[\pi-\arg \left(e^{i \arccos x}-\varphi(\zeta)\right)\right]\right\}=\cos \kappa_{j, n} .
\end{gathered}
$$

First we consider the situation $a=1$. In this case the expressions in 13 become

$$
x_{j, n}=\cos \kappa_{j, n}, \quad j=1, \ldots, n, \quad n \in \Lambda .
$$

The nodes are close to the zeros of the Chebyshev polynomials. That's why the term corresponding to the $\sigma$ 's influence in (13) vanishes when $a=1$.

Let us analyze now the case $a=1 / 2$. We consider the following identities

$$
\cos \left[\pi-\arg \left(e^{i \arccos x_{j, n}}-\varphi(\zeta)\right)\right]=\frac{\varphi(\zeta)-x_{j, n}}{\sqrt{\varphi^{2}(\zeta)-2 \varphi(\zeta) x_{j, n}+1}}
$$

and

$$
\sin \left[\pi-\arg \left(e^{i \arccos x_{j, n}}-\varphi(\zeta)\right)\right]=\frac{\sqrt{1-x_{j, n}^{2}}}{\sqrt{\varphi^{2}(\zeta)-2 \varphi(\zeta) x_{j, n}+1}} .
$$

Substituting (15) and (16) in (13) we arrive at the quadratic equations:

$$
x_{j, n}^{2}-\frac{2 \sin ^{2} \kappa_{j, n}}{\varphi(\zeta)} x_{j, n}+\frac{\sin ^{2} \kappa_{j, n}}{\varphi^{2}(\zeta)}-\cos ^{2} \kappa_{j, n}=0, \quad j=1, \ldots, n, \quad n \in \Lambda
$$

For each $j=1, \ldots, n, n \in \Lambda$ we obtained the following solutions

$$
x_{j, n}=\frac{1}{\varphi(\zeta)}\left[\sin ^{2} \kappa_{j, n}+\cos \kappa_{j, n} \sqrt{\varphi^{2}(\zeta)-\sin ^{2} \kappa_{j, n}}\right] .
$$

During the process of finding these above solutions we introduce some extra solutions that we removed. Observe that when $\zeta$ tends to $\infty$ the expressions in 17 reduce to (14). This is in accordance with the fact that $\widetilde{\sigma}$ approaches $\lambda_{0}$ as $\zeta \rightarrow \infty$, see (11), hence we only considered the positive branch of the square root in (17).

Finally take $a=0$. From (13) we have that

$$
\begin{gathered}
x_{j, n} \cos \left\{2\left[\pi-\arg \left(e^{i \arccos x}-\varphi(\zeta)\right)\right]\right\} \\
-\sqrt{1-x_{j, n}^{2}} \sin \left\{2\left[\pi-\arg \left(e^{i \arccos x}-\varphi(\zeta)\right)\right]\right\}=\cos \kappa_{j, n} .
\end{gathered}
$$


We use the conditions (15) and (16), and obtain the following expression

$$
x_{j, n}=\frac{2 \zeta \cos \kappa_{j, n}}{\varphi(\zeta)+2 \cos \kappa_{j, n}}, \quad j=1, \ldots, n, \quad n \in \Lambda .
$$

Taking into account that $\varphi(\zeta)=\zeta+\sqrt{\zeta^{2}+1}$ we see that the above expression is reduced to (14) when $\zeta$ goes to infinity.

\section{Connection with orthogonal polynomials}

Let $\mu$ be a positive finite Borel measure with infinitely many points in its support $\operatorname{supp}(\mu)$. Set $\Delta$ denoting the least interval which contains $\operatorname{supp}(\mu)$. A collection of monic polynomials $\left\{q_{\mu, n}\right\}_{n \in \mathbb{Z}_{+}}, \mathbb{Z}_{+}=\{0,1, \ldots\}$ is the family orthogonal polynomials with respect to $\mu$ if its elements satisfy the following orthogonality relations

$$
0=\int x^{v} q_{\mu, n}(x) \mathrm{d} \mu(x), \quad v=0,1, \ldots, n-1, \quad n \in \mathbb{Z}_{+} .
$$

Each $q_{\mu, n}$ has $n$ single roots lying in the interior of $\Delta$ (we denote $\stackrel{\circ}{\Delta}$ ) such that it vanishes at most once in each interval of $\Delta \backslash \operatorname{supp}(\mu)$ (see [4, Theorem 5.2] or [7. Chapter 1]). We also know that $q_{\mu, n+1}$ and $q_{\mu, n}$ interlace their zeros. In [20] B. Wendroff proved that given two polynomials $P_{n}$ and $P_{n+1}$, with $\operatorname{deg} P_{n+1}=$ $\operatorname{deg} P_{n}+1=n+1$, that interlace zeros, there always exist measures $\mu$ such that $P_{n}=q_{\mu, n}$ and $P_{n+1}=q_{\mu, n+1}$. Now we find some of these measures.

We say then a polynomial $P_{n}(x)=\prod_{j=1}^{n}\left(x-x_{j}\right)$ of degree $n$ is admissible with respect to the measure $\mu$, if its roots are all simple, lying in $\stackrel{\circ}{\Delta}$, with at most one zero into each interval of $\Delta \backslash \operatorname{supp}(\mu)$. The system of nodes $\left(x_{1}, \ldots, x_{n}\right)$ is also said to be admissible with respect to $\mu$.

Lemma 1. Let $P_{n}(x)=\prod_{j=1}^{n}\left(x-x_{j}\right)$ and $\widetilde{P}_{n}(x)=\prod_{j=1}^{n-1}\left(x-\widetilde{x}_{j}\right)$ be two admissible polynomials with respect to $\mu$ that satisfy $x_{1}<\widetilde{x}_{1}<x_{2}<\cdots<\widetilde{x}_{n-1}<x_{n}$. Then there exists a positive integrable function $\rho_{n}$ with respect to $\mu$ ( $\rho_{n}$ is a weight function for $\mu$ ) such that for the measure $\mu_{n}$ which differential form $d \mu_{n}(x)=\rho_{n}(x) d \mu(x)$, $x \in \operatorname{supp}(\mu), P_{n} \equiv q_{\mu_{n}, n}$ and $\widetilde{P}_{n} \equiv q_{\mu_{n}, n-1}$ are the $n$-th and $n-1$-th monic orthogonal polynomials with respect to $\mu_{n}$, respectively.

In the proof we follow techniques used in [12].

Proof. Consider $\Phi$ a set of weight functions such that for every constant $\alpha>0$ it satisfies: 
i) $\rho \in \Phi \Longrightarrow \alpha \rho \in \Phi$.

ii) $(\rho, \widetilde{\rho}) \in \Phi^{2}=\Phi \times \Phi \Longrightarrow \alpha \rho+(1-\alpha) \widetilde{\rho} \in \Phi, \alpha \leq 1$.

iii)If a polynomial $Q$ satisfies $\int Q(x) \rho(x) \mathrm{d} \mu(x)>0$ for all $\rho \in \Phi$, then $Q \geq 0$ in $\operatorname{supp}(\mu)$.

Two examples of sets of weight functions satisfying the above conditions are the positive polynomials and positive simple functions in [16, Definition 1.16]. In general, the positive linear combinations of a Chevyshev system (see [11, Chapter II]) conform a set as $\Phi$. Examples of Chevyshev systems can be found in [15] (also in [9]).

Given $\rho \in \Phi$ we set

$$
\begin{aligned}
& \mathbf{v}_{\rho}=\left(\int \widetilde{P}_{n}(x) \rho(x) \mathrm{d} \mu(x), \ldots, \int x^{n-2} \widetilde{P}_{n}(x) \rho(x) \mathrm{d} \mu(x),\right. \\
& \left.\int P_{n}(x) \rho(x) \mathrm{d} \mu(x), \ldots, \int x^{n-1} P_{n}(x) \rho(x) \mathrm{d} \mu(x)\right) \in \mathbb{R}^{2 n-1} .
\end{aligned}
$$

Let us focus on $\mathcal{K}=\left\{\mathbf{v}_{\rho}: \rho \in \Phi\right\}$. Proving Lemma 1 reduces to showing that $\mathcal{K}$ contains the origin. From condition $(i)$ we have that the origin belongs to $\mathcal{K}$ 's closure, $\overline{\mathcal{K}}$. Since $\mathcal{K}$ is open we need to prove the origin is an interior point. We proceed by contradiction. Suppose that the origin belongs to the boundary of $\mathcal{K}$. This is $\mathbf{O} \in \partial \mathcal{K}=\bar{K} \backslash \mathcal{K}$. There exists a hyper-plane $\mathcal{A}$ that touches tangentially $\partial \mathcal{K}$ at $\mathbf{O}$. On the other hand we have that condition (ii) implies that $\mathcal{K}$ is convex, then there exists a vector $\mathbf{a}=\left(a_{0, n-1}, \ldots, a_{n-2, n-1}, a_{0, n}, \ldots, a_{n-1, n}\right)$ which is orthogonal with respect to $\mathcal{A}$ in the sense of the standard inner vector product $(\mathbf{a} \cdot \mathbf{u}=0$, for all $\mathbf{u} \in \mathcal{A})$, and for each $\mathbf{v}_{\rho} \in \mathcal{K}, \mathbf{v}_{\rho} \cdot \mathbf{a}>0$. So the polynomials

$$
p_{n-1}(x)=a_{0, n-1}+a_{1, n-1} x+\ldots+a_{n-2, n-1} x^{n-2}
$$

and

$$
p_{n}(x)=a_{0, n}+a_{1, n} x+\ldots+a_{n-1, n} x^{n-1}
$$

satisfy that

$$
0<\int\left[p_{n-1}(x) \widetilde{P}_{n}(x)+p_{n} P_{n}(x)\right] \rho(x) \mathrm{d} \mu(x), \quad \text { for all } \quad \rho \in \Phi .
$$

According to condition (iii) the polynomial $\mathcal{P}(x)=p_{n-1}(x) \widetilde{P}_{n}(x)+p_{n} P_{n}(x)$, with real coefficients, must be non-negative in $\operatorname{supp}(\mu)$. However we shall prove that this is impossible, arriving then to a contradiction.

Assume that $\mathcal{P}(x)=p_{n-1}(x) \widetilde{P}_{n}(x)+p_{n} P_{n}(x)$ does not change sign in $\operatorname{supp}(\mu)$. Suppose that there is a point $t \in \operatorname{supp}(\mu)$, such that $t=x_{k} k \in\{1, \ldots, n\}$ satisfying that $\mathcal{P}\left(x_{k}\right)=0$, then taking into account that $\widetilde{P}_{n}$ and $P_{n}$ interlace zeros, we have that $p_{n-1}\left(x_{k}\right)=0$. Also, since $\mathcal{P}$ is non-negative on $\operatorname{supp}(\mu)$, we have that $t=x_{k}$ is a zero of multiplicity even for $\mathcal{P}$. Consider $S=\left\{t_{1}, \ldots, t_{\ell}\right\} \subset\left\{x_{1}, \ldots, x_{n}\right\}$ the set of all points where $P_{n}$ and $\mathcal{P}$ vanishe at same time. Then we can write 


$$
\mathcal{P}=q(x) \prod_{i=1}^{\ell}\left(x-t_{i}\right)^{2 d_{i}}, \quad d_{i} \in \mathbb{N}, \quad i=1, \ldots, \ell,
$$

where $q$ is a polynomial with positive values at every root of $P_{n}$. We also write

$$
p_{n-1}(x)=\widetilde{p}(x) \prod_{i=1}^{\ell}\left(x-t_{i}\right) .
$$

The polynomial $\widetilde{p}$ has degree $\operatorname{deg} p_{n}-\ell$. Since $\widetilde{P}_{n}$ and $P_{n}$ interlace zeros, we have that

$$
\frac{\widetilde{P}_{n}(x)}{P_{n}(x)}=\sum_{j=1}^{n} \frac{\lambda_{j}}{x-x_{j}}, \quad \lambda_{j}>0, \quad j=1, \ldots, n .
$$

Observe that

$$
\frac{\prod_{i=1}^{\ell}\left(x-t_{i}\right)}{P_{n}(x)}=\frac{1}{\prod_{i=1}^{\ell}\left(x-t_{i}\right)^{2 d_{i}-1} q(x)}\left[p_{n-1}(x) \sum_{j=1}^{n} \frac{\lambda_{j}}{x-x_{j}}+p_{n}(x)\right] .
$$

This means that the above function satisfies that

$$
\frac{1}{\prod_{i=1}^{\ell}\left(z-t_{i}\right)^{2 d_{i}-1} q(z)}\left[p_{n-1}(z) \sum_{j=1}^{n} \frac{\lambda_{j}}{z-x_{j}}+p_{n}(z)\right]=O\left(\frac{1}{z^{n-\ell}}\right) \quad \text { as } \quad z \rightarrow \infty,
$$

which is a holomorphic functions on $\overline{\mathbb{C}} \backslash\left(\left\{x_{1}, \ldots, x_{n}\right\} \backslash S\right)$. For each $v=0, \ldots, n-$ $\ell-2$ we have then

$$
\frac{z^{v}}{\prod_{i=1}^{\ell}\left(z-t_{i}\right)^{2 d_{i}-1} q(z)}\left[p_{n-1}(z) \sum_{j=1}^{n} \frac{\lambda_{j}}{z-x_{j}}+p_{n}(z)\right]=O\left(\frac{1}{z^{2}}\right) \quad \text { as } \quad z \rightarrow \infty
$$

also holomorphic functions on $\overline{\mathbb{C}} \backslash\left(\left\{x_{1}, \ldots, x_{n}\right\} \backslash S\right)$. Set the elements $y_{j} \in$ $\left\{x_{1}, \ldots, x_{n}\right\} \backslash S, j=1, \ldots, n-\ell$ with $y_{1}<y_{2}<\cdots<y_{n-\ell}$, and $\widetilde{\lambda}_{j}, j=1, \ldots, n-\ell$ the coefficients $\lambda$ 's defined in 21) corresponding to points $y_{j}$. Also let $\lambda_{j}^{\prime}$ denote the $\lambda$ 's of $t_{j}, j=1, \ldots, \ell$. Call $F$ the set of the roots of the polynomial $q$ defined in (19). Consider a closed integration path $\Gamma$ with winding number 1 for all its interior points. Denote $\operatorname{Ext}(\Gamma)$ and $\operatorname{Int}(\Gamma)$ the unbounded and bounded connected components respectively of the complement of $\Gamma$. Take $\Gamma$ so that $I \subset \operatorname{Int}(\Gamma)$ and $F \subset \operatorname{Ext}(\Gamma)$. From Cauchy's Theorem and the above two conditions, it follows that 


$$
\begin{aligned}
& 0=\frac{1}{2 \pi i} \int_{\Gamma} \frac{z^{v}}{\prod_{i=1}^{\ell}\left(z-t_{i}\right)^{2 d_{i}-1} q(z)}\left[p_{n-1}(z) \sum_{j=1}^{n} \frac{\lambda_{j}}{z-x_{j}}+p_{n}(z)\right] \mathrm{d} z \\
& =\frac{1}{2 \pi i} \int_{\Gamma} \frac{z^{v} p_{n-1}(z) \sum_{j=1}^{n-\ell} \frac{\tilde{\lambda}_{j}}{z-y_{j}} \mathrm{~d} z}{\prod_{i=1}^{\ell}\left(z-t_{i}\right)^{2 d_{i}-1} q(z)}+\frac{1}{2 \pi i} \int_{\Gamma} \frac{z^{v} p_{n}(z) \mathrm{d} z}{\prod_{i=1}^{\ell}\left(z-t_{i}\right)^{2 d_{i}-1} q(z)} .
\end{aligned}
$$

Since $\frac{z^{v} p_{n}(z)}{\ell} \in \mathcal{H}(\operatorname{Int}(\Gamma))$ the second term vanishes. From $[20$, using

$$
\prod_{i=1}^{\ell}\left(z-t_{i}\right)^{2 d_{i}-1} q(z)
$$

the Cauchy integral formula, we obtain:

$$
0=\sum_{j=1}^{n-\ell} y_{j}^{v} \widetilde{p}\left(y_{j}\right) \frac{\tilde{\lambda}_{j}}{\prod_{i=1}^{\ell}\left(y^{j}-t_{i}\right)^{2\left(d_{i}-1\right)} q\left(y_{j}\right)}=0, \quad v=0, \ldots, n-\ell-1 .
$$

Taking into account that for each $j=1, \ldots, n-\ell, \frac{\widetilde{\lambda}_{j}}{\prod_{i=1}^{\ell}\left(y^{j}-t_{i}\right)^{2\left(d_{i}-1\right)} q\left(y_{j}\right)}>0$, we conclude that the above orthogonality relations imply that $\widetilde{p}$ must change sign at least $n-\ell$ times, hence $\operatorname{deg} \widetilde{p} \geq n-\ell$. Since $\operatorname{deg} \widetilde{p}=\operatorname{deg} p_{n-1}-\ell \leq n-\ell-1$ we arrive at a contradiction which completes the proof.

Consider a monic polynomial $P_{n}(x)=\prod_{j=1}^{n}\left(x-x_{j}\right)$ with degree $n \in \mathbb{N}$ which is $\mu$ admissible. We say that a weight function $\rho_{n}$ on $\operatorname{supp}(\mu)$ is orthogonal with respect to $P_{n}(x)$ and $\mu$ if $P_{n} \equiv q_{\mu_{n}, n}$, where $\mathrm{d} \mu_{n}(x)=\rho_{n}(x) \mathrm{d} \mu(x), x \in \operatorname{supp}(\mu)$. We also say that $\rho_{n}$ is orthogonal with respect to $\mathbf{x}_{n}=\left(x_{1}, \ldots, x_{n}\right)$ and $\mu$. A sequence of weight functions $\left\{\rho_{n}\right\}_{n \in \mathbb{N}}$ is a family of orthogonal weight functions with respect to the sequence of polynomials $\left\{P_{n}\right\}_{n \in \mathbb{N}}$, if for each $n \in \mathbb{N}, P_{n} \equiv q_{\mu_{n}, n}$.

Let $q_{m(n)}$ be an arbitrary polynomial with degree $\operatorname{deg} q_{m(n)}(x)=2 n-m(n)-1$ being positive on $[-1,1]$. Let $\mu_{n}$ denote the measure with differential form $\mathrm{d} \mu_{n}(x)=q_{m(n)}^{-1}(x) \mathrm{d} \mu(x), x \in \operatorname{supp}(\mu)$. Set a system of nodes $\mathbf{x}_{n}=\left(x_{1}, \ldots, x_{n}\right)$ such that $P_{n}(x)=\prod_{j=1}^{n}\left(x-x_{j}\right)=q_{\mu_{n}, n}$. This means that $\mathbf{x}_{n}$ is the system of $n$ nodes corresponding to the Gaussian quadrature rule for the measure $\mu_{n}$. Given an arbitrary polynomial $p \in \Pi_{m(n)}$, we have that 


$$
q_{m(n)}(x) p(x)-\sum_{j=1}^{n} q_{m(n)}\left(x_{j}\right) p\left(x_{j}\right) L_{j, n}(x)=q_{\mu_{n}, n}(x) \mathcal{P}_{n-1}(x)
$$

where $L_{j, n}(x):=\prod_{\substack{k=1 \\ k \neq j}}^{n} \frac{x-x_{k}}{x_{j}-x_{k}}, \quad j=1, \ldots, n$, and $\mathcal{P}_{n-1}$ is a certain polynomial with $\operatorname{deg} \mathcal{P}_{n-1}=n-(m(n)-\operatorname{deg} p)-1 \leq n-1$.

Observe that $\int p(x) \mathrm{d} \mu(x)=\int q_{m(n)}(x) p(x) \mathrm{d} \mu_{n}(x)$. Hence from 22 we obtain $\int p(x) \mathrm{d} \mu(x)-\int \sum_{j=1}^{n} q_{m(n)}\left(x_{j}\right) p\left(x_{j}\right) L_{j, n}(x) \mathrm{d} \mu_{n}(x)=\int q_{\mu_{n}, n}(x) \mathcal{P}_{n-1}(x) \mathrm{d} \mu_{n}(x)$,

which vanishes because $q_{\mu_{n}, n}$ satisfies the orthogonality relations for $\mu_{n}$ as in (18). We conclude then

$$
\int p(x) \mathrm{d} \mu(x)=\sum_{j=1}^{n} p\left(x_{j, n}\right) q_{m(n)}\left(x_{j, n}\right) \int L_{j, n}(x) \frac{\mathrm{d} \lambda_{0}(x)}{q_{m(n)}(x)}=\sum_{j=1}^{n} w_{j, n} p\left(x_{j, n}\right) .
$$

This is an interpolatory integration rule with degree of exactness $m(n)$, where the weights can be defined via

$$
w_{j, n}=q_{m(n)}\left(x_{j}\right) \int L_{j, n}(x) \mathrm{d} \mu_{n}(x)=q_{m(n)}\left(x_{j}\right) \widetilde{w}_{j, n}, \quad j=1, \ldots, n .
$$

The numbers $\widetilde{w}_{j, n}, j=1, \ldots, n$ are the weights corresponding to a Gaussian quadrature rule, which are all positive. Since $q_{m(n)}$ is also positive the weights $w_{j, n}>0$. According to Pólya's condition a sequence of these rules of integration is convergent.

Let us consider $\mathbf{x}=\left\{\mathbf{x}_{n}=\left(x_{1, n}, \ldots, x_{n, n}\right)\right\}_{n \in \mathbb{N}}$ an admissible scheme of nodes for a measure $\mu$, and take a corresponding family of orthogonal weights $\left\{\rho_{n}\right\}_{n \in \mathbb{N}}$. For each $n, \mu_{n}$ denotes the measure with differential form $\mathrm{d} \mu(x)=\rho_{n}(x) \mathrm{d} \mu(x)$, and introduce its family of orthonormal polynomials $\left\{p_{\mu_{n}, j}\right\}_{j \in \mathbb{Z}_{+}}$. This means that $p_{\mu_{n}, j} \equiv q_{\mu_{n}, j} /\left\|q_{\mu_{n}, j}\right\|_{2, \mu_{n}}, j \in \mathbb{Z}_{+}$where $\|\cdot\|_{2, \mu_{n}}$ denotes the $\mathrm{L}_{2}$ norm corresponding to the measure $\mu_{n}$.

Given a function $f \in \mathrm{L}_{2, \mu_{n}}$ and $j \in \mathbb{Z}_{+}$we consider the $j$-th partial sum of the Fourier series corresponding to $f / \rho_{n}$ on the bases $\left\{p_{\mu_{n}, j}\right\}_{j \in \mathbb{Z}_{+}}$:

$$
S_{f, \mu_{n}, j}=\sum_{k=0}^{j-1} f_{k} p_{\mu_{n}, k}(x), \quad f_{k}=\int f(x) p_{\mu_{n}, k}(x) \mathrm{d} \mu_{n}(x), \quad k=0, \ldots, j-1 .
$$

Using the Christoffel-Darboux identity (see [19, Theorem 3.2.2]) we can deduce

$$
S_{f, \mu_{n}, j}(x)=\int \frac{q_{\mu_{n}, j}(x) q_{\mu_{n}, j-1}(t)-q_{\mu_{n}, j}(t) q_{\mu_{n}, j-1}(x)}{\left\|q_{\mu_{n}, j-1}\right\|_{2, \mu_{n}}^{2}(x-t)} f(t) \mathrm{d} \mu_{n}(t) .
$$


The following result is an extension of [19, Theorem 15.2.4 (equality 15.2.7)]

Lemma 2. Let $\left(x_{1}, \ldots, x_{n}\right)$ be an $\mu$ admissible system of nodes. Given a polynomial $q_{m(n)}$ take the system of weights $\left(w_{1, n}, \ldots, w_{n, n}\right)$ whose elements $w_{j, n}, j=1, \ldots, n$, are constructed using (23). Then there always exists a weight $\rho_{n}$ such that

$$
\frac{w_{j, n}}{q_{m(n)}\left(x_{j}\right)}=\frac{\left\|q_{\tau_{n}, n-1}\right\|_{2, \tau_{n}}^{2} S_{1 / \rho_{n}, \tau_{n}, n}\left(x_{j}\right)}{q_{\tau_{n}, n-1}\left(x_{j}\right) q_{\tau_{n}, n}^{\prime}\left(x_{j}\right)}=-\frac{\left\|q_{\tau_{n}, n}\right\|_{2, \tau_{n}}^{2} S_{1 / \rho_{n}, \tau_{n}, n+1}\left(x_{j}\right)}{q_{\tau_{n}, n+1}\left(x_{j}\right) q_{\tau_{n}, n}^{\prime}\left(x_{j}\right)},
$$

where the measure $\tau_{n}$ is such that $\frac{d \tau_{n}}{d \mu}=\frac{\rho_{n}}{q_{m(n)}}$. Thus sign $w_{j, n}=\operatorname{sign} S_{1 / \rho_{n}, \tau_{n}, n}\left(x_{j}\right)=$ sign $S_{1 / \rho_{n}, \tau_{n}, n+1}\left(x_{j}\right), j=1, \ldots, n$.

Proof. Take an orthogonal weight $\rho_{n}$ with respect to the system of $n$ nodes $\left(x_{1}, \ldots, x_{n}\right)$ and the measure with differential form $\mathrm{d} \mu(x) / q_{m(n)}(x)$. According to (23) and taking into account that $P_{n} \equiv q_{\mu_{n}, n}$ where the measure $\tau_{n}$ has the differential form $\mathrm{d} \tau_{n}(x)=\rho_{n}(x) \mathrm{d} \mu(x) / q_{m(n)}(x)$, we have the following

$$
w_{j, n}=q_{m(n)}\left(x_{j}\right) \int \frac{q_{\tau_{n}, n}(x)}{q_{\tau_{n}, n}^{\prime}\left(x_{j}\right)\left(x-x_{j}\right)} \frac{d \mu(x)}{q_{m(n)}(x)}, \quad j=1, \ldots, n .
$$

Arranging the above formula and using the identity 24) we obtain that

$$
\begin{gathered}
w_{j, n}=\frac{q_{m(n)}\left(x_{j}\right)\left\|q_{\tau_{n}, n}\right\|_{2, \tau_{n}}^{2}}{q_{\tau_{n}, n+1}\left(x_{j}\right) q_{\tau_{n}, n}^{\prime}\left(x_{j}\right)} \int \frac{q_{\tau_{n}, n+1}\left(x_{j}\right) q_{\tau_{n}, n}(x)}{\left\|q_{\tau_{n}, n}\right\|_{2, \tau_{n}}^{2}\left(x-x_{j}\right)} \frac{1}{\rho_{n}(x)} \frac{\rho_{n}(x) d \mu(x)}{q_{m(n)}(x)} \\
=-\frac{q_{m(n)}\left(x_{j}\right)\left\|q_{\tau_{n}, n}\right\|_{2, \tau_{n}}^{2}}{q_{\tau_{n}, n+1}\left(x_{j}\right) q_{\tau_{n}, n}^{\prime}\left(x_{j}\right)} S_{1 / \rho_{n}, \tau_{n}, n}\left(x_{j}\right),
\end{gathered}
$$

which proves the second identity in (25). Since $q_{\tau_{n}, n+1}\left(x_{j}\right) q_{\tau_{n}, n}^{\prime}\left(x_{j}\right)<0, \quad j=$ $1, \ldots, n$, then $\operatorname{sign} w_{j, n}=\operatorname{sign} S_{1 / \rho_{n}, \tau_{n}, n+1}\left(x_{j}\right)$. Following the above steps we can prove the first equality in (25) and sign $w_{j, n}=\operatorname{sign} S_{1 / \rho_{n}, \tau_{n}, n}\left(x_{j}\right)$.

The following two results are consequences of the above Lemma2

Lemma 3. An admissible scheme of nodes $\mathbf{x}=\left\{\mathbf{x}_{n}=\left(x_{1, n}, \ldots, x_{n, n}\right)\right\}_{n \in \mathbb{N}}$ is convergent if there exists a family of orthogonal weights $\left\{\rho_{n}\right\}_{n \in \mathbb{N}}$ with respect to $\mathbf{x}$ and the sequence of measures $\left\{d \tau_{n}(x)=d \mu(x) / q_{m(n)}(x)\right\}_{n \in \mathbb{N}}$ satisfying

$$
\lim _{n \rightarrow \infty}\left\|1-\rho_{n}(x) S_{1 / \rho_{n}, \tau_{n}, n}(x)\right\|_{[-1,1], \infty}=0,
$$

where $\|\cdot\|_{[-1,1], \infty}$ denotes the supremum norm on $[-1,1]$.

Proof. Assuming the equality (26), there exists a number $N>0$ such that for every $n \geq N$ the function $S_{1 / \rho_{n}, \mu_{n}, n}(x)>0$ on $[-1,1]$ particularly at the nodes. According to Lemma2 the coefficients $w_{j, n}, j=1, \ldots, n$, are also positive. This completes the proof. 
Lemma 4. Consider the varying measure $d \mu_{n}(x)=d \mu(x) / q_{m(n)}(x)$ and their orthogonal polynomials $q_{\mu_{n}, n}(x)=\prod_{j=1}^{n}\left(x-x_{j, n}\right)$ and $q_{\mu_{n}, n-1}(x)=\prod_{j=1}^{n-1}\left(x-x_{j, n-1}\right)$, $n \in \mathbb{N}$. Let $\mathbf{y}=\left\{\mathbf{y}_{n}=\left(y_{1, n}, \ldots, y_{n, n}\right)\right\}_{n \in \mathbb{N}}$ be a scheme of nodes such that for each $n \in \mathbb{N}$

$$
-1<y_{1, n}<x_{1, n-1}<y_{2, n}<\cdots<x_{n-1, n-1}<y_{n, n}<1 .
$$

Assume that the polynomials $P_{n}(x)=\prod_{j=1}^{n}\left(x-y_{j, n}\right), \quad n \in \mathbb{N}$ satisfy

$$
\lim _{n \rightarrow \infty} \frac{1}{\left\|q_{\mu_{n}, n-1}\right\|_{2, \mu_{n}}^{2}}\left[\frac{\left(q_{\mu_{n}, n}-P_{n}\right) q_{\mu_{n}, n-1}}{q_{m(n)}^{2}}\right]^{\prime}=0, \quad \text { on }[-1,1] .
$$

Then $\mathbf{y}$ is convergent.

Proof. From Lemma 1 we ensure the existence of a weight function $\rho_{n}$ such that the polynomials $q_{\tau_{n}, n-1}$ and $P_{n}$ belong to the family of orthogonal polynomials corresponding to the measure $\rho_{n}(x) \mathrm{d} \mu(x) / q_{m(n)}(x)$. Let us analyze the function

$$
\begin{aligned}
& \left|1-\frac{\left\|P_{n}\right\|_{2, \rho_{n} d \mu / q_{m(n)}}^{2}}{q_{m(n)}(x)\left\|q_{\mu_{n}, n-1}\right\|_{2, \mu_{n}}^{2}} S_{1 / \rho_{n}, \rho_{n} d \mu / q_{m(n), n}}(x)\right| \\
& =\frac{1}{q_{m(n)}(x)}\left|S_{q_{m(n)}, \mu_{n}, n}(x)-\frac{\left\|\widetilde{P}_{n}\right\|_{2, \rho_{n} d \mu / q_{m(n)}}^{2}}{\left\|q_{\mu_{n}, n-1}\right\|_{2, \mu_{n}}^{2}} S_{1 / \rho_{n}, \rho_{n} d \mu / q_{m(n), n}}(x)\right|
\end{aligned}
$$

We have used that $S_{q_{m(n)}, \mu_{n}, n} \equiv q_{m(n)}$, hence we need to show that

$$
\lim _{n \rightarrow 0} \frac{1}{q_{m(n)}(x)}\left|S_{1 / \rho_{n}, \mu_{n}, n}(x)-\frac{\left\|\widetilde{P}_{n}\right\|_{2, \rho_{n} d \mu / q_{m(n)}}^{2}}{\left\|q_{\mu_{n}, n-1}\right\|_{2, \mu_{n}}^{2}} S_{1 / \rho_{n}, \rho_{n} d \mu / q_{m(n), n}}(x)\right|=0 .
$$

Applying (24) we observe that

$$
\begin{aligned}
& \frac{1}{q_{m(n)}(x)}\left(S_{1 / \rho_{n}, \mu_{n}, n}(x)-\frac{\left\|\widetilde{P}_{n}\right\|_{2, \rho_{n} d \mu / q_{m(n)}}^{2}}{\left\|q_{\mu_{n}, n-1}\right\|_{2, \mu_{n}}^{2}} S_{1 / \rho_{n}, \rho_{n} d \mu / q_{m(n), n}(x)}\right)=\frac{q_{m(n)}^{-1}(x)}{\left\|q_{\mu_{n}, n-1}\right\|_{2, \mu_{n}}^{2}} \\
& \times \int\left(\frac{q_{\mu_{n}, n}(x) q_{\mu_{n}, n-1}(t)-q_{\mu_{n}, n}(t) q_{\mu_{n}, n-1}(x)}{x-t}-\frac{P_{n}(x) q_{\mu_{n}, n-1}(t)-P_{n}(t) q_{\mu_{n}, n-1}(x)}{x-t}\right)
\end{aligned}
$$




$$
\times \frac{\mathrm{d} \mu(t)}{q_{m(n)}(t)}
$$

Let us consider the kernel

$$
\begin{gathered}
\frac{q_{\mu_{n}, n}(x) q_{\mu_{n}, n-1}(t)-q_{\mu_{n}, n}(t) q_{\mu_{n}, n-1}(x)}{\left\|q_{\mu_{n}, n-1}\right\|_{2, \mu_{n}}^{2} q_{m(n)}(x) q_{m(n)}(t)(x-t)}-\frac{P_{n}(x) q_{\mu_{n}, n-1}(t)-P_{n}(t) q_{\mu_{n}, n-1}(x)}{\left\|q_{\mu_{n}, n-1}\right\|_{2, \mu_{n}}^{2} q_{m(n)}(x) q_{m(n)}(t)(x-t)} \\
=\frac{\left(q_{\mu_{n}, n}-P_{n}\right)(x) q_{\mu_{n}, n-1}(t)-\left(q_{\mu_{n}, n}-P_{n}\right)(t) q_{\mu_{n}, n-1}(x)}{\left\|q_{\mu_{n}, n-1}\right\|_{2, \mu_{n}}^{2} q_{m(n)}(x) q_{m(n)}(t)(x-t)}=\mathcal{K}(x, t) .
\end{gathered}
$$

From Taylor's Theorem we obtain that

$$
\mathcal{K}(x, t)=\frac{1}{\left\|q_{\mu_{n}, n-1}\right\|_{2, \mu_{n}}^{2}}\left[\frac{\left(q_{\mu_{n}, n}-P_{n}\right) q_{\mu_{n}, n-1}}{q_{m(n)}^{2}}\right]^{\prime}(s)
$$

for some $s$ in between of $x$ and $t$, so the assumption (28) completes the proof.

\section{Asymptotic analysis}

Let us consider the varying measure $\mu_{n}$ with $\mathrm{d} \mu_{n}(x) / \mathrm{d} x=\left(q_{m(n)}(x) \sqrt{1-x^{2}}\right)^{-1}$, where $q_{m(n)}(x)=q_{\kappa} \frac{1-a}{k} n(x)=\left(\prod_{k=1}^{\kappa}\left(x-\zeta_{j}\right)\right)^{2 \frac{1-a}{k} n}, \quad n \in \Lambda$. Let $\sigma$ be the zero counting measure of $q_{k}$. This is $\sigma=\frac{1}{\kappa} \sum_{k=1}^{k} \delta_{\zeta_{k}}$. Set the analytic logarithmic potential corresponding to the measure $\sigma$ :

$$
g(z, \sigma)=-\int \log (z-\zeta) \mathrm{d} \sigma(\zeta)
$$

We take the logarithmic branch such that $g(z, \sigma)$ is analytic on a domain $D \subset K$ that contains the interval $[-1,1]$, and also for every $x \in[-1,1]$,

$$
V^{\sigma}(x)=\int \log \frac{1}{|x-\zeta|} \mathrm{d} \sigma(\zeta)=g(x, \sigma)=-\int \log (z-\zeta) \mathrm{d} \sigma(\zeta) .
$$

Since $\sigma$ is symmetric we $\int \arg (x-\zeta) \mathrm{d} \sigma(\zeta)=0$. In each compact $K \subset D$ we have that $\frac{1}{2 n} \log \frac{1}{q_{m(n)}(z)}=(1-a) g(z, \sigma)$ on $K$.

Lemma 5. Let $d \mu_{n}(x) / d x=\left(q_{m(n)}(x) \sqrt{1-x^{2}}\right)^{-1}, n \in \mathbb{N}$ be a sequence of measures as above. Then 


$$
q_{\mu_{n}, n}=\left(1+O\left(e^{-c n}\right)\right) \exp \left\{-n V^{\bar{\nu}}\right\} K_{1, n}+O\left(e^{-c n}\right) \exp \left\{-n V^{\bar{\nu}}\right\} K_{2, n}
$$

and

$$
\frac{d_{n, n-1}}{2^{2 n a}} q_{\mu_{n}, n-1}=\left(1+O\left(e^{-c n}\right)\right) \exp \left\{-n V^{\bar{\nu}}\right\} K_{2, n}+O\left(e^{-c n}\right) \exp \left\{-n V^{\bar{\nu}}\right\} K_{1, n}
$$

where $d_{n, n-1}=-\left(2 \pi i\left\|q_{\mu_{n}, n-1}\right\|_{\mu_{n}, 2}^{2}\right)^{-1}$

$$
K_{1, n}(x)=2 \cos n\left((1-a) \pi \int_{x}^{1} d \sigma(t)-a \arccos x\right)
$$

and

$$
K_{2, n}(x)=\frac{1}{i} \cos n\left((1-a) \pi \int_{x}^{1} d \sigma(t)-(a-1 / n) \arccos x\right) .
$$

Proof. We study a matrix Riemann-Hilbert problem like in [14, Theorem 2.4] whose solution $Y$ is a $2 \times 2$ matrix function satisfying the following conditions:

1. $Y \in \mathcal{H}(\mathbb{C} \backslash[-1,1])$ (all the entries of $Y$ are analytic on $\mathbb{C} \backslash[-1,1]$ ),

2. $Y_{+}(x)=Y_{-}(x)\left(\begin{array}{c}1\left(q_{m(n)}(x) \sqrt{1-x^{2}}\right)^{-1} \\ 0\end{array}\right), x \in(-1,1)$,

3. $Y(z)\left(\begin{array}{cc}z^{-n} & 0 \\ 0 & z^{n}\end{array}\right)=\mathbb{I}+O(1 / z)$ as $z \rightarrow \infty, \mathbb{I}$ is the $2 \times 2$ identity matrix.

4. $Y(z)=O\left(\begin{array}{c}1|z \pm 1|^{-1 / 2} \\ 1|z \pm 1|^{-1 / 2}\end{array}\right)$ as $z \rightarrow \mp 1$.

According to [14, Theorem 2.4] (see also [13]) the $Y$ solution of above matrix Riemann-Hilbert problem (for short Y-RHP) is unique and has the form

$$
Y(z)=\left(\begin{array}{cc}
q_{\mu_{n}, n}(z) & -\frac{1}{2 \pi i} \int \frac{q_{\mu_{n}, n}(x)}{z-x} \mathrm{~d} \mu_{n}(x) \\
d_{n, n-1} q_{\mu_{n}, n-1}(z) & -\frac{d_{n, n-1}}{2 \pi i} \int \frac{q_{\mu_{n}, n-1}(x)}{z-x} \mathrm{~d} \mu_{n}(x)
\end{array}\right) .
$$

The key of our procedure follows the ideas introduced in [1]. We find a relationship between $Y$ and the matrix solution $R: \mathbb{C} \backslash \gamma \rightarrow \mathbb{C}^{2 \times 2}$ corresponding to another Riemann-Hilbert problem (R-RHP) for a closed Jordan curve $\gamma$ positively oriented surrounding the interval $[-1,1]$ :

1. $R \in \mathcal{H}(\mathbb{C} \backslash \gamma)$,

2. $R_{+}(\zeta)=R_{-}(\zeta) V_{n}(\zeta), \zeta \in \gamma$, with $V_{n} \in \mathcal{H}(D)$,

3. $R(z) \rightarrow \mathbb{I}$ as $z \rightarrow \infty$, 
where $V_{n}=\mathbb{I}+O\left(\varepsilon^{n}\right)$ with $0 \leq \varepsilon<1$, uniformly on compact subsets of $K$ as $n \rightarrow \infty$. Those conditions imply that $R=\mathbb{I}+O\left(\varepsilon^{n}\right)$ uniformly on $\mathbb{C}$ as $n \rightarrow \infty$. There is a chain of transformations to arrive from $Y$ to $R$, which we represent $Y \rightarrow T \rightarrow S \rightarrow R$. Once we have arrived to $R$, we recover the entries of $Y$ going back from $R$ to $Y$.

From [3], Corollary 4] we have that the zero counting measures $v_{n}$ defined in (8) corresponding to the monic orthogonal polynomials $q_{\mu_{n}, n}(z)=\prod_{j=1}^{n}\left(z-x_{j, n}\right)$ with respect to the varying measures $\mu_{n}$, satisfy

$$
v_{n} \stackrel{\star}{\rightarrow} \bar{v}=(1-a) \widetilde{\sigma}+a \lambda_{0} \quad \text { as } \quad n \rightarrow \infty,
$$

where $\widetilde{\sigma}$ denotes the balayage of the measure $\sigma$ out of $\mathbb{C} \backslash[-1,1]$ onto $[-1,1]$.

The measure $\bar{v}$ is the so called (see [17, Theorem I.1.3]) equilibrium measure under the influence of the external field $(1-a) V^{\sigma}(z)$. From (35) we have the following equilibrium condition

$$
V^{\bar{\nu}}(t)-(1-a) V^{\sigma}(t)=a V^{\lambda_{0}}(t)=a \log 2, \quad t \in[-1,1] .
$$

Observe the conditions (3) in both Riemann Hilbert problems. $Y$ requires a normalization at infinity to get to $R$ 's behavior at infinity. We modify $Y$ to obtain a Riemann-Hilbert problem whose solution is defined on the same set as $Y$, which approaches $\mathbb{I}$ as $n \rightarrow \infty$. Let us introduce the function $g(z, \bar{v})$, which is the analytic potential corresponding to the measure $\bar{v}$ described in (35)

$$
g(z, \bar{v})=-\int \log (z-t) \mathrm{d} \bar{v}(t)=V^{\bar{v}}(z)-i \int \arg (z-t) \mathrm{d} \bar{v}(t),
$$

with arg denoting the principal argument $g(z, \bar{v}) \in \mathcal{H}(K \backslash(-\infty, 1])$. Substituting $g(z, \bar{v})$ in (36) we obtain

$$
g_{+}(x, \bar{v})+g_{-}(x, \bar{v})-2 a \log 2-2(1-a) g(x, \sigma)=0, \quad x \in[-1,1] .
$$

and

$$
g_{-}(x, \bar{v})-g_{+}(x, \bar{v})= \begin{cases}0 & \text { if } x \geq 1 \\ 2 \pi i & \text { if } x \leq 1 \\ 2 \wp(x) & \text { if } x \in(-1,1)\end{cases}
$$

with

$$
\wp(x)=\pi i \int_{x}^{1} \mathrm{~d} \bar{v}(t)=\pi i\left[(1-a) \int_{x}^{1} \mathrm{~d} \widetilde{\sigma}(t)-\frac{a}{\pi} \arccos (x)\right] .
$$

Consider the matrices $G(z)=\left(\begin{array}{cc}e^{n g(z, \bar{v})} & 0 \\ 0 & e^{-n g(z, \bar{v})}\end{array}\right)$ and $L=\left(\begin{array}{cc}2^{n a} & 0 \\ 0 & 2^{-n a}\end{array}\right)$. We define the matrix function $T=L Y G L^{-1}$. So $T$ is the unique solution of the following Riemann-Hilbert problem (T-RHP)

1. $T \in \mathcal{H}(\mathbb{C} \backslash[-1,1])$,

2. $T_{+}(x)=T_{-}(x) M(x), x \in(-1,1)$, 
3. $T(z)=\mathbb{I}+O(1 / z)$ as $z \rightarrow \infty$,

4. $T(z)=O\left(\begin{array}{l}1|z \pm 1|^{-1 / 2} \\ 1|z \pm 1|^{-1 / 2}\end{array}\right)$ as $z \rightarrow \mp 1$,

where according to (38) and (39) the jump matrix $M(x)=\left(\begin{array}{cc}e^{-2 n \wp(x)} & \left(1-x^{2}\right)^{-1 / 2} \\ 0 & e^{2 n \wp(x)}\end{array}\right)$, $x \in(-1,1)$.

According to [6, Theorem 1.34] there exists a domain $D$ containing the interval $[-1,1]$ where the function $\wp$ in $(40)$ admits an analytic extension on $D \backslash[-\infty, 1]$ as

$$
\mathcal{A}(z)=\pi i \int_{z}^{1} \mathrm{~d} \bar{v}(\zeta)=\pi i \int_{z}^{1} \bar{v}^{\prime}(\zeta) \mathrm{d} \zeta
$$

where $\bar{v}^{\prime}(\zeta)=\frac{\psi(\zeta)}{\sqrt{1-\zeta^{2}}}$, with $\psi \in \mathcal{H}(D)$ and $\psi(x)>0, x \in(-1,1)$. Observe that $\mathcal{A}_{+}(x)=\wp(x)=-\mathcal{A}_{-}(x)$, then we write $M(x)=\left(\begin{array}{cc}e^{-2 n \mathcal{H}_{+}(x)} & \left(1-x^{2}\right)^{-1 / 2} \\ 0 & e^{-2 n \mathcal{A}_{-}(x)}\end{array}\right)$.

We now seek jump conditions as we have in R-RHP. Consider a closed Jordan curve $\gamma \in D$ surrounding $[-1,1]$ as we have in R-RHP. Let $\Omega$ denote the bounded connected component of $\mathbb{C} \backslash \gamma$. We consider the function $\sqrt{z^{2}-1} \in \mathcal{H}(\mathbb{C} \backslash[-1,1]$ with ${\sqrt{x^{2}-1}}_{ \pm}= \pm i \sqrt{1-x^{2}}, x \in(-1,1)$. We introduce the matrix function $S$ as follows

$$
S(z)=\left\{\begin{array}{cl}
T(z) & \text { when } z \in \mathbb{C} \backslash(\gamma \cup \Omega) \\
T(z)\left(\begin{array}{cc}
1 \\
-i \sqrt{z^{2}-1} e^{-2 n \mathcal{A}(z)} & 1
\end{array}\right) & \text { when } z \in \Omega
\end{array} .\right.
$$

The matrix function $S$ is the solution of the following Riemann-Hilbert problem (S-RHP):

1. $S \in \mathcal{H}(\mathbb{C} \backslash(\gamma \cup[-1,1]))$,

2. $S_{+}(x)=S_{-}(x)\left(\begin{array}{cc}0 & \left(1-x^{2}\right)^{-1 / 2} \\ -\left(1-x^{2}\right)^{1 / 2} & 0\end{array}\right)$, when $x \in(-1,1)$ and

$$
S_{+}(\zeta)=S_{-}(\zeta)\left(\begin{array}{cc}
1 & 0 \\
-i \sqrt{z^{2}-1} e^{-2 n \mathcal{H}(z)} & 1
\end{array}\right) \text {, when } \zeta \in \gamma \text {. }
$$

3. $S(z)=\mathbb{I}+O(1 / z)$ as $z \rightarrow \infty$,

4. $S(z)=O\left(\begin{array}{l}1|z \pm 1|^{-1 / 2} \\ 1|z \pm 1|^{-1 / 2}\end{array}\right)$ as $z \rightarrow \mp 1$.

The jump matrix on $\gamma$ approaches uniformly the identity matrix $I$. However it does not happen in $[-1,1]$. We fix this problem in the interval following the steps in [13]. Consider the matrix

$$
N(z)=\left(\begin{array}{ll}
\frac{a(z)+a^{-1}(z)}{2} \frac{D(\infty)}{D(z)} & \frac{a(z)-a^{-1}(z)}{2 i} D(\infty) D(z) \\
\frac{a(z)-a^{-1}(z)}{-2 i} \frac{1}{D(\infty) D(z)} & \frac{a(z)+a^{-1}(z)}{2} \frac{D(z)}{D(\infty)}
\end{array}\right),
$$


where $D(z)=\left(\frac{z}{\sqrt{z^{2}-1}}+1\right)^{1 / 2}, \quad D(\infty)=\sqrt{2}$ and $a(z)=\frac{(z-1)^{1 / 4}}{(z+1)^{1 / 4}}$. Hence $N$ is the solution of the following Riemann-Hilbert problem

1. $N \in \mathcal{H}(\mathbb{C} \backslash[-1,1])$,

2. $N_{+}(x)=N_{-}(x)\left(\begin{array}{cc}0 & \left(1-x^{2}\right)^{-1 / 2} \\ -\left(1-x^{2}\right)^{1 / 2} & 0\end{array}\right), x \in(-1,1)$,

3. $N(z)=\mathbb{I}+O(1 / z)$ as $z \rightarrow \infty$,

4. $N(z)=O\left(\begin{array}{l}1|z \pm 1|^{-1 / 2} \\ 1|z \pm 1|^{-1 / 2}\end{array}\right)$ as $z \rightarrow \mp 1$.

Introduce the matrix function $R(z)=S(z) N^{-1}$. Taking into account that $R$ and $S$ satisfy the same jump conditions across $(-1,1)$ we have that $R_{+}(x)=R_{-}(x)$. So $R \in \mathcal{H}(\mathbb{C} \backslash(\gamma \cup\{-1,1\}))$. Since $\operatorname{det} N=1$ and from (42) we have that

$$
N^{-1}(z)=O\left(\begin{array}{cc}
|z \pm 1|^{-1 / 2}|z \pm 1|^{-1 / 2} \\
1 & 1
\end{array}\right) \quad \text { as } \quad z \rightarrow \mp 1 .
$$

Thus, when $z \rightarrow \mp 1$

$$
R(z)=O\left(\begin{array}{l}
1|z \pm 1|^{-1 / 2} \\
1|z \pm 1|^{-1 / 2}
\end{array}\right) O\left(\begin{array}{cc}
|z \pm 1|^{-1 / 2} & |z \pm 1|^{-1 / 2} \\
1 & 1
\end{array}\right) .
$$

This implies

$$
R(z)=O\left(\begin{array}{l}
|z \pm 1|^{-1 / 2}|z \pm 1|^{-1 / 2} \\
|z \pm 1|^{-1 / 2}|z \pm 1|^{-1 / 2}
\end{array}\right)
$$

which means that each entry of $R$ has isolated singularities at $z=-1$ and $z=1$ with $R(z)=O|z \pm 1|^{-1 / 2}$ as $z \rightarrow \mp 1$, and they are removable. So $R$ satisfies the following Riemann-Hilbert conditions:

1. $R \in \mathcal{H}(\mathbb{C} \backslash \gamma)$,

2. $R_{+}(\zeta)=R_{-}(\zeta)\left(\begin{array}{cc}1 & 0 \\ e^{-2 n \mathcal{A}(\zeta)} & 1\end{array}\right)$, when $\zeta \in \gamma$.

3. $R(z)=\mathbb{I}+O(1 / z)$ as $z \rightarrow \infty$.

From (41) $2 \mathcal{A} \in \mathcal{H}(D \backslash[-\infty, 1])$ and $\mathcal{R} e\left(2 \mathcal{A}_{ \pm}(x)\right)=0, x \in[-1,1]$. Using the fact $2 \mathcal{A}_{ \pm}^{\prime}(x)= \pm 2 i \bar{v}^{\prime}(x)=\mp 2 \pi i \frac{\psi(x)}{\sqrt{1-x^{2}}}, x \in(-1,1)$ and the Cauchy-Riemann conditions we have that $\frac{\partial \mathcal{R} e\left(2 \mathcal{A}_{ \pm}\right)}{\partial y}(x)>0, x \in[-1,1]$. Since $\mathcal{R} e(2 \mathcal{A})$ is a harmonic function on $D \backslash[-1,1]$ we have $\operatorname{Re}(2 \mathcal{A}(z))>0, z \in D \backslash[-1,1]$. So given an arbitrary compact set $K \subset D \backslash[-1,1]$ there exists a constant $c(K)>0$ and an $N \in \mathbb{N}$ large enough such that for every $n \geq N$ the function $\operatorname{Re}(2 \mathcal{A}(z))(z))>c(K), z \in K$ and $n \geq N$. Note also that $\phi_{n} \rightarrow 0$ as $n \rightarrow \infty$. So according [1] we arrive at $R(z)=\mathbb{I}+O\left(e^{-c n}\right)$ uniformly as $n \rightarrow \infty$ for each compact set $K \subset \mathbb{C} \backslash[-1,1]$. Take $z \in \operatorname{Int}(\gamma)$. Going back now from $R$ to $Y$, and considering just the first column, we have that: 


$$
\begin{gathered}
e^{n g(z, \bar{v})}\left(\begin{array}{l}
q_{\mu_{n}, n}(z) \\
2^{-2 n a} d_{n, n-1} q_{\mu_{n}, n-1}(z)
\end{array}\right)=\left(\mathbb{I}+O\left(e^{-c n}\right)\right) \\
\times\left(\begin{array}{ll}
\frac{a(z)+a^{-1}(z)}{2} \frac{D(\infty)}{D(z)} & \frac{a(z)-a^{-1}(z)}{2 i} D(\infty) D(z) \\
\frac{a(z)-a^{-1}(z)}{-2 i} \frac{1}{D(\infty) D(z)} & \frac{a(z)+a^{-1}(z)}{2} \frac{D(z)}{D(\infty)}
\end{array}\right)\left(\begin{array}{c}
1 \\
\left(1-z^{2}\right)^{1 / 2} e^{-2 n \mathcal{A}(z)}
\end{array}\right) .
\end{gathered}
$$

Take the + boundary values of all quantities involved when $z \rightarrow x \in(-1,1)$. Using the following identities from [13] or [14]

$$
\frac{a_{+}(x) \pm a_{+}(x)}{2}=\frac{1}{\sqrt{2}\left(1-x^{2}\right)^{1 / 4}} \exp \left( \pm \frac{i}{2} \arccos x \mp i \frac{\pi}{4}\right)
$$

we have $\exp \left\{n V^{\bar{\nu}}(x)\right\}\left(\begin{array}{l}q_{\mu_{n}, n}(x) \\ 2^{-2 n a} d_{n, n-1} q_{\mu_{n}, n-1}(x)\end{array}\right)=\left(\mathbb{I}+O\left(e^{-c n}\right)\right)\left(\begin{array}{l}K_{1, n}(x) \\ K_{2, n}(x)\end{array}\right)$, where $K_{2, n}(x)=\frac{1}{i} \cos n\left((1-a) \pi \int_{x}^{1} \mathrm{~d} \widetilde{\sigma}(t)-(a-1 / n) \arccos x\right)$ and

$$
K_{1, n}(x)=2 \cos n\left((1-a) \pi \int_{x}^{1} \mathrm{~d} \widetilde{\sigma}(t)-a \arccos x\right) .
$$

Finally we obtain

$$
q_{\mu_{n}, n}(x)=\left(1+O\left(e^{-c n}\right)\right) e^{-n V^{\bar{v}}(x)} K_{1, n}(x)+O\left(e^{-c n}\right) e^{-n V^{\bar{v}}(x)} K_{2, n}(x)
$$

and

$$
\frac{d_{n, n-1}}{2^{2 n a}} q_{\mu_{n}, n-1}(x)=\left(1+O\left(e^{-c n}\right)\right) e^{-n V^{\bar{v}}(x)} K_{2, n}(x)+O\left(e^{-c n}\right) e^{-n V^{\bar{v}}(x)} K_{1, n}(x),
$$

which are exactly the equalities stated in (31) and 32).

\section{Proof of Theorem 1}

We combine Lemma 5 and Lemma 28 First we choose a special scheme of nodes $\mathbf{y}=\left\{\mathbf{y}_{n}=\left(y_{1, n}, \ldots, y_{n, n}\right)\right\}_{n \in \Lambda}$ which satisfies (10). The corresponding polynomials have the following form

$$
P_{n}(x)=\prod_{j=1}^{n}\left(x-y_{j, n}\right)=\Phi_{n}(x) \cos n\left((1-a) \pi \int_{x}^{1} \mathrm{~d} \widetilde{\sigma}(t)-a \arccos x\right),
$$

Where $\Phi_{n}$ is a real valued function on $[-1,1]$ that never vanishes. Let us rewrite the relation (31) as follows

$$
q_{\mu_{n}, n}(x)=2 \exp \left\{-n V^{\bar{\nu}}(x)\right\}\left(\cos n\left((1-a) \pi \int_{x}^{1} \mathrm{~d} \widetilde{\sigma}(t)-a \arccos x\right)+O\left(e^{-c n}\right)\right)
$$




$$
=2 \exp \left\{-n V^{\bar{v}}(x)\right\} \cos n\left((1-a) \pi \int_{x}^{1} \mathrm{~d} \widetilde{\sigma}(t)-a \arccos x+O\left(e^{-c n}\right)\right) .
$$

Combining the above equality with (43) we obtain that $\int_{x_{j, n}}^{y_{j, n}} \mathrm{~d} \bar{v}(t)=O\left(e^{-c n}\right)$ and $x_{j, n}-y_{j, n}=O\left(e^{-c n}\right)$. This implies that $\limsup _{n \rightarrow \infty}\left|q_{\mu_{n}, n}-P_{n}\right|^{1 / n}(x)=\exp \left(-c-V^{\bar{v}}(x)\right)$ on $[-1,1]$. Hence

$$
\limsup _{n \rightarrow \infty}\left(\frac{1}{\left\|q_{\mu_{n}, n-1}\right\|_{2, \mu_{n}}^{2}}\left[\frac{\left(q_{\mu_{n}, n}-P_{n}\right) q_{\mu_{n}, n-1}}{q_{m(n)}^{2}}\right]^{\prime}\right)^{1 / n}(x)=\exp \left(-c+V^{\sigma}(x)\right)<1 .
$$

Here we have taken into account that $\operatorname{dist}\left(\left\{\zeta_{1}, \ldots, \zeta_{K}\right\},[-1,1]\right)>1$, which yields $V^{\sigma}(x)<0, x \in[-1,1]$. Then we see that condition (28) in Lemma 4 is satisfied. We now prove that condition (27) holds.

Taking into account the equality (32) we have that the zeros of the polynomials $q_{\mu_{n}, n-1}$ satisfy that for each $j=1, \ldots, n-1, n \in \mathbb{N}$

$$
(1-a) \pi \int_{x_{j, n-1}}^{1} \mathrm{~d} \widetilde{\sigma}(t)-(a-1 / n) \arccos x+O\left(e^{-c n}\right)=\frac{2 j-1}{2 n} \pi .
$$

For each $j=1, \ldots, n-1$, we subtract the above equality (44) to (10), and we obtain that

$$
\int_{y_{j, n}}^{x_{j, n-1}} \mathrm{~d} \bar{v}(t)=\frac{1}{n}(1+o(1)) \quad \text { as } \quad n \rightarrow \infty .
$$

This means that for $n$ large enough $y_{j, n}<x_{j, n-1}, j=1, \ldots, n-1$. Considering now the $j$ th equality in (10) and the $j+1$ th in (44) we have that

$$
\int_{x_{j, n-1}}^{y_{j+1, n}} \mathrm{~d} \bar{v}(t)=\frac{1}{n}(\pi-1+o(1)) \quad \text { as } \quad n \rightarrow \infty
$$

which implies that $x_{j, n-1}<y_{j+1, n}$. So condition 27] holds. This proves that the scheme $\mathbf{y}$ is convergent.

Once we know that $\mathbf{y}$ is convergent, we can construct another convergent scheme $\mathbf{x}=\left\{\mathbf{x}_{n}=\left(x_{1, n}, \ldots, x_{n, n}\right)\right\}_{n \in \Lambda}$ taking

$$
x_{j, n}-y_{j, n} \leq A e^{-\ell n}, \quad j=1, \ldots, n, \quad n \in \Lambda,
$$

and follow the previous process. This completes the proof.

\section{References}

1. A.I. Aptekarev and W. Van Assche. Scalar and matrix Riemann-Hilbert approach to the strong asymptotics of Padé approximants and complex orthogonal polynomials with vaying 
weight. J. Approx. Theory 129 (2004) 129-166.

2. T. Bloom, D.S. Lubinsky, H. Stahl. What distribution of points are possible for convergent sequences of interpolatory integration rules. Constr. Appr. Theo. 9, (1993), 41-58.

3. T. Bloom, D.S. Lubinsky, H. StAhL. Interpolatory integration rules and orthogonal polynomials with varying weights. Numer. Algorithms. 3, (1992), 55-66.

4. T. S. Chinara. An itroduction to orthogonal polynomials. Gordon and Breach, Science Publishers, Inc. New York, London, Paris.

5. P. J. Davis, P. Rabinowitz. Methods of Numerical Integration, 2nd Edition, San Diego CA: Academic Press (1984).

6. P. Deift, T. Kriecherbauer, K. T.-R. McLaughlin. New results on the equilibrium measures for logarithmic potentials in presence of an external field. J. of Approx. Theory. 95, (1998), 388-475.

7. G. Freud. Orthogonal polynomials. Pergamon Press London, Toronto, New York (1971).

8. U. Fidalgo, A. López, G. López Lagomasino, and V.N. Sorokin. Mixed type multiple orthogonal polynomials for two Nikishin systems. Constr. Approx. 32 (2010) 255-306.

9. U. Fidalgo, S. Medina Peralta, J. Mínguez Cenicero. Mixed type multiple orthogonal polynomials: Perfectness and interlacing properties. Linear Algebra Appl. 438 (2013), 12291239.

10. F.D. Gaknov. Boundary Values Problems. Oxford-New York: Pergamon Press (1966).

11. M. G. Krein and A. A. Nudel'man. The Markov Moment Problem and Extremal Problems. Transl. Math. Monogr., Vol. 50, Amer. Math. Soc., Providence, R. I., 1977.

12. A. Kroó, D. Schmidt And M. Sommer. On some properties of A-spaces and their relation to Hobby-Rice theorem. J. of Approx. Th. 68 (1999), 136-141.

13. A.B.J. KuiJlaARs. Riemann-Hilbert analysis for orthogonal polynomials, in: E. Koelink, W. VAN Assche (EDs), Orthogonal polynomials and special functions, in: Lect. Notes Math. 1817, Springer-Verlag, Berlin, (2003), 167-210.

14. A.B.J. Kuijlaars, K.T.-R. Mc Laughlin, W. Van Assche, and M. Vanlessen. The RiemannHilbert approach to strong asymptotics for orthogonal polynomials on $[-1,1]$. Adv. Math. 188 (2004), 337-398.

15. E.M. Nikishin. On simultaneous Padé approximants. Matem. Sb. 113 (1980), 499-519 (Russian); English translation in Math. USSR Sb. 41 (1982), 409-425.

16. W. Rudin, Real and Complex Analysis, 3nd ed., McGraw-Hill, New York, 1986.

17. E.B. SAfF And V. Totiк. Logarithmic Potentials with External Fields. New York NY: Series of Comprehensive Studies in Mathematics, Vol. 316, Springer (1997).

18. H. Stahl and V. Totik. General Orthogonal Polynomials. Cambridge UK: Cambridge University Press (1992).

19. G. Szegő. Orthogonal Polynomials. New York NY: Vol. XXIII, Coll. Pub. Amer. Math. Soc. (1939).

20. B. Wendroff. On orthogonal polynomials, Proc. Amer. Math. Soc. 12 (1961) 554-555. 